\title{
Investigation on dependence of the electron's characteristic energy on the similarity variable in diffusion-recombination dominated glow discharge in pure gases using average degree of ionization as parameter
}

\author{
M. Aram ${ }^{1}$, N. Morshedian' ${ }^{2}$ A. Mehramiz ${ }^{3}$ and S. Behrouzinia ${ }^{1^{*}}$ \\ Photonics and Quantum Technologies Research School, Nuclear Science and Technology Research Institute, \\ Tehran, $\operatorname{Iran}^{1}$ \\ Plasma and Nuclear Fusion Research School, Nuclear Science and Technology Research Institute, Tehran, Iran ${ }^{2}$ \\ Physics Department, Science Faculty, Imam Khomeini International University, Qazvin, Iran ${ }^{3}$
}

Received: 09-May-2020; Revised: 25-June-2020; Accepted: 27-June-2020

(C)2020 M. Aram et al. This is an open access article distributed under the Creative Commons Attribution (CC BY) License, which permits unrestricted use, distribution, and reproduction in any medium, provided the original work is properly cited.

\begin{abstract}
Through the continuity equation of electrons and positive ions for the radial electron density variation with a timeindependent glow discharge in pure media, the diagram of the characteristic energy of the electrons versus similarity variable in glow discharge has been derived, while the linear terms of diffusion and nonlinear term of recombination have been considered in the model. The characteristic curves for three gases of $\mathrm{CO}_{2}, \mathrm{~N}_{2}$, and Helium at constant temperature, and three different degrees of ionization have been derived. It has shown that in the degrees of ionization of $10^{-3}$, the curve has been strongly shifted to the high electron temperature, i.e., glow discharge in highly ionized plasmas, which still low electron temperature has been forbidden by recombination process. The effect of gas properties on the electron temperature in constant similarity variable, has been discussed; also, the difference of the gas flow and gas sealed of glow discharge vessels respect to the similarity variable has been studied.
\end{abstract}

\section{Keywords}

Glow discharge, Diffusion-recombination, Characteristic diagram.

\section{Introduction}

The physical aspects of the plasma environment and its applications, which is the main idea of gaseous electronics and gas-discharge based devices like gas lasers, have been specifically investigated in the past decades, and there are some good general references in this field $[1-3]$. The physics of the glow discharge mechanism, which is regular in the pumping method of gas lasers $[4,5]$ and glow discharge tubes of the spectra-chemical analysis, have been investigated [6, 7], but it is still under more investigations [8-13]. The most important factor in the subject of statistical physics of the plasma and gas-discharge media is the electron energy distribution function (E.E.D.F), which can be resulted generally by solving of the Boltzmann equation [14, 15]. Using this function and the excitation cross-section of the atomic and/or molecular levels, the excitation rate of the level can be calculated, and the most important parameter, which determined E.E.D.F is the electron temperature (Te).

*Author for correspondence

113
In fact, Te of the electrical discharge is related to reduced electric field $(\mathrm{E} / \mathrm{N})$ while, it can be shown that there is a relation between the and similarity variable of discharge (NR), which in cylindrical geometry, is production of the total number density of media $(\mathrm{N})$ by the inner radius of the discharge vessel. The mathematical form of the relationship is complicated and obtained from the solution of the equation, which relates the $T_{e}$ and $N R$ under the boundary condition $\left(\mathrm{n}_{\mathrm{e}}\left(\mathrm{R}_{0}\right)=0\right)$ in the glow discharge at cylindrical geometry. So, the geometrical show of the relation, or characteristic diagram of the glow discharge, is more familiar [2-4]. As has been reported previously [16], by using a semi-analytical physical model, some basic parameters of plasma media can be roughly estimated. In this work, by continuing that line in the study, the characteristics of the gas-discharge plasma, the results of including the nonlinear term of recombination in the model have been presented for the positive column of a low flow longitudinal continuous glow discharge tube, using the continuity of the equation and the E.E.D.F of Maxwell-Boltzmann. According to the above- 
mentioned methods, the diagram of Te versus the product of the total particle number density by tube inner radius (NR) in a diffusion-dominated process have been presented. In final step, the Te versus NR diagram under a more realistic condition based on the diffusion-recombination dominated regime has been obtained, where the average electron density $\left(n_{e}\right)$ is presented as the minor parameter, and the results have been discussed.

In a global view the objective of this paper is related to the investigation on the kinetic of the glow discharge of gases, especially the spectroscopic ally, as more as possible in detailed. The important note is that although the used method is limited to the pure gases but it can be used very simply for the mixture of gases, albeit the highly electronegative gases needs more attention.

\section{Theoretical preliminaries}

All formulas have been calculated in S.I units, and the laboratory reference frame has been used for obtaining the relations. The flow velocity of the gas mixture has been taken low, and its effect on the electrical properties of discharge media can be ignored, although it may still affect the thermal and laser properties of the media. In such conditions and by combining the basic formulas of the timeindependent diffusion-recombination dominated continuity the equation for electrons and ions, the following formula for electron number density will be obtained as follows [2, 3]:

$$
\nabla^{2} \mathbf{n}_{\mathbf{e}}-\left(\frac{\mathbf{v}_{\text {eff }}}{\mathbf{D}_{\mathbf{a}}}\right) \mathbf{n}_{\mathrm{e}}=\mathbf{S}
$$

Where, $v_{\text {eff }}=\left(v_{\text {ion }}-v_{\text {att }}-v_{\text {rec }}\right)$ is the effective ionization-recombination frequency and is the result of the combination of the ionization ( $\left.v_{i o n}\right)$, attachment $\left(v_{a t t}\right)$ and recombination $\left(v_{r e c}\right)$ frequencies in the glow discharge media (the effect of attachment has been ignored in following as the first estimation). The recombination frequency is defined as $v_{r e c}=\gamma N_{+}$where $\gamma$ is the (radiantly) recombination rate. The other parameter, am-bipolar diffusion constant $D_{a}\left(=\frac{\mu_{e} D_{+}+\mu_{+} D_{e}}{\mu_{e}+\mu_{+}}\right)$, is defined by using the diffusion constants of positive ions ( $D_{+}$ ), and electrons $\left(D_{e}\right)$ and the mobilities of positive ions $\left(\mu_{+}\right)$and electrons $\left(\mu_{e}\right)$. The $\mathrm{S}$ term is related to the effective external source or sinks, so it has 114 been ignored (i.e. $\mathrm{S}=0$ ), here. Using the Einstein's relation $\left(\mathrm{D}_{q} / \mu_{q}=\mathrm{kT}_{q} / \mathrm{q}\right)$ and solving the radial part of the equation (1) at cylindrical coordinate with appropriate boundary conditions $\left(n_{e}(0)=n_{0}\right.$ and $\left.n_{e}(r=R)=0\right)$, the following characteristic equation of steady-state glow discharge in gases has been derived as follows :

$$
\mathbf{N}_{\text {tot }}^{2}\left[\left(\mathbf{R}_{\text {ion, rec }}\right)\left(\frac{1}{\left(\boldsymbol{\mu}_{\mathbf{e}}\right)}+\frac{1}{\left(\boldsymbol{\mu}_{+}\right)}\right)_{R D}\right]=\frac{\left(\mathbf{k} \mathbf{T}_{\mathbf{e}}+\mathbf{k} \mathbf{T}_{+}\right)}{\mathbf{e}}\left(\frac{2.405}{\mathbf{R}}\right)^{2}
$$

Where $\boldsymbol{k T}_{\boldsymbol{e}}$ and $\boldsymbol{k} \boldsymbol{T}_{+}$are the characteristic thermal energy of electrons and ions, respectively, $N_{t o t}$ is the total number of particle density, and $R$ is the inner radius of the tube, respectively. Because of extracting total number density of particles $\left(N_{t o t}\right)$, all of frequencies are replaced by rates as follows:

$\left[R_{x}\right]=\left\langle\sigma_{x} v_{e}\right\rangle$

Where $\boldsymbol{R}_{x}$ and $\sigma_{x}$ are the ionization or recombination rates and cross-section, respectively, and $v_{e}=\sqrt{\frac{2 E_{e}}{m_{e}}}$ is electron thermal velocity. So, the effective ionization-recombination rate, $\left(R_{\text {ion,rec }}\right)$, has been defined by including both of ionization and recombination effects in the model, as follows:

$R_{\text {ion }, \text { rec }}=\mathrm{R}_{\text {ion }}+D . I \times \boldsymbol{\gamma}$

Where $D . I=\overline{n_{e}} / N$ and $\gamma$ are the (averaged) degree of ionization of the media and the recombination rate, respectively. The dimension of $R_{i o n, r e c}$ is the same as the ionization rate, obviously.

The next important quantities in formula (2) are $\left[1 /\left(\boldsymbol{\mu}_{e}\right)\right]_{\boldsymbol{R} D}$ and $\left[1 /\left(\boldsymbol{\mu}_{+}\right)\right]_{\boldsymbol{R} D}$ which are reduced reverse of the reduced mobility of the electron and positive ion, respectively where each one is defined as follows:

$$
\begin{aligned}
& {\left[1 /\left(\mu_{e}\right)\right]_{R D}=e\left(\frac{1}{m_{e}}\right)\left\langle\left(\sigma_{e}^{c}\right) v_{e}\right\rangle} \\
& {\left[1 /\left(\mu_{+}\right)\right]_{R D}=\left[1 /\left(\mu_{+}\right)\right]_{R D}=e\left(\frac{2}{M_{+}}\right)\left\langle\sigma_{+}^{c} v_{+}\right\rangle}
\end{aligned}
$$

Where, in (5), $\left(\boldsymbol{\sigma}_{e}^{c}\right)$ and $\boldsymbol{v}_{\boldsymbol{e}}$ are electron collision cross-section, and thermal velocity of the electron, respectively. In (6), $\boldsymbol{M}_{+}, \quad \boldsymbol{\sigma}_{+}^{c}$ and $\boldsymbol{v}_{+}$are (molecular/atomic) mass of the positive ion, ionmedia collision cross-section, and velocity of positive 
ion relative to the media, respectively. Two main parameters that were needed to calculate all terms in relation (4) are the cross-sections and the distribution function. The cross-sections have been provided by curve fitting to experimental data (The ionization cross-sections) and/or by a simple theoretical model (The recombination cross-section). The MaxwellBoltzmann is used as a reasonable approximation for the distribution function of electrons (using $\mathrm{T}_{\mathrm{e}}$ ) and, or ions (using $\mathrm{T}_{\mathrm{i}}=\mathrm{T}_{\text {gas }}$ ). The ionization cross-section of the helium, $\mathrm{CO}_{2}$, and $\mathrm{N}_{2}$ from threshold energy value up to $5 \mathrm{Kev}$ and $10 \mathrm{Kev}$ which is given by [17] have been imported to MATLAB (MathWorks ${ }^{\circledR}$ ), and the best formula for fitting the data has been found as the relation (7). The results are shown in Figure 1 (a, $\mathrm{b}$, and $\mathrm{c})$.

$\sigma_{\text {ioniz }}(E)=A\left(\sigma_{\max }, E_{\max }\right) \frac{a E^{2}+b E}{c E^{3}+d E^{2}+f} g$

Where $\mathrm{E}$ is electron kinetic energy and $\left(\sigma_{\max }, E_{\max }\right)$ are dependent on the maximum value of cross-section and its electron energy. The ionization rates are calculated according to the relation (3) by numerical integration from the threshold ionization energies, i.e., $26^{\mathrm{ev}}, 13.77^{\mathrm{ev}}$, and $15.58 \mathrm{ev}$ for helium, $\mathrm{CO}_{2}$, and $\mathrm{N}_{2}$, respectively, up to the $10^{\mathrm{kev}}$ (for helium) and $5^{\mathrm{kev}}$ (for $\mathrm{CO}_{2} \& \mathrm{~N}_{2}$ ). The parameters for helium, $\mathrm{CO}_{2}$, and $\mathrm{N}_{2}$ are given in Table 1 as follows:

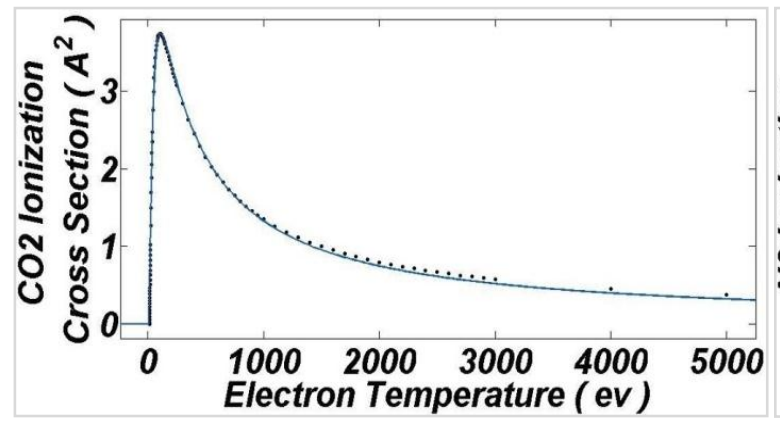

(a)

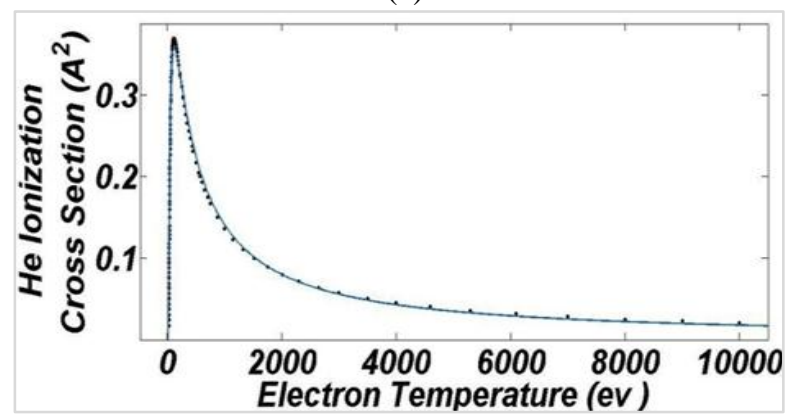

(c)

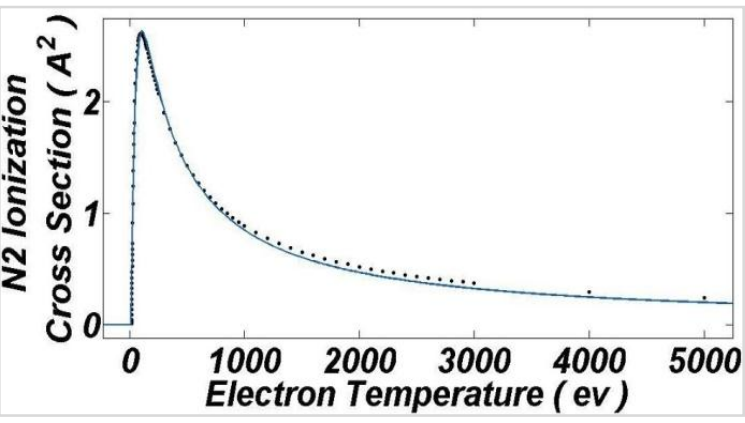

(b)

Figure 1 The total ionization cross-section of (a) CO2 (b) N2 (c) helium

Table 1 The parameters of the ionization cross-section formula for $\mathrm{CO} 2, \mathrm{~N} 2$ and helium

\begin{tabular}{lllllll}
\hline & $\mathrm{a}$ & $\mathrm{b}$ & $\mathrm{c}$ & $\mathrm{d}$ & $\mathrm{f}$ & $\mathrm{g}$ \\
\hline helium & 0.06586 & -1.742 & $5.918 \mathrm{e}^{-4}$ & 0.1661 & 4.522 & 1.664 \\
\hline $\mathrm{CO}_{2}$ & 0.07886 & -0.744 & $2.951 \mathrm{e}^{-6}$ & $8.02 \mathrm{e}^{-4}$ & 1.183 & 0.06372 \\
\hline $\mathrm{N}_{2}$ & 0.1508 & -0.4633 & $9.609 \mathrm{e}^{-7}$ & $2.227 \mathrm{e}^{-4}$ & 0.524 & $6.686 \mathrm{e}^{-3}$ \\
\hline
\end{tabular}

In the process of handling the mobility, part of the formula (2), because the electron mobility is twice as high as the ionic mobility in magnitude in any media, the reverse of total reduced electron mobility, $\left[1 /\left(\mu_{\mathrm{e}}\right)\right]_{\boldsymbol{R} D}$, is reasonably ignored compare to the reverse of total reduced (positive) ions mobility, $\left[1 /\left(\boldsymbol{\mu}_{+}\right)\right]_{\boldsymbol{R} \boldsymbol{D}}[18]$, the experimental data of the ionic mobility have been used where ionic motility of the $\mathrm{CO}_{2}, \mathrm{~N}_{2}$, and the helium has been shown in Table 2 . 
Table 2 Reduced ion mobility's $\left(10^{-4} \mathrm{~m}^{2} \cdot \mathrm{v}^{-1} \cdot \mathrm{s}^{-1}\right)$ in $273 \mathrm{~K}$ and $1.103 \mathrm{~Pa}$

\begin{tabular}{lll}
\hline $\mathrm{CO}_{2}{ }^{+} / \mathrm{CO}_{2}$ & $\mathrm{~N}_{2}{ }^{+} / \mathrm{N}_{2}$ & $\mathrm{He}^{+} / \mathrm{He}$ \\
\hline $1.26[19]$ & $1.78(300 \mathrm{~K})[20]$ & $10.4(300 \mathrm{~K})[21]$ \\
\hline
\end{tabular}

Here, $\left[1 /\left(\boldsymbol{\mu}_{+}\right)\right]_{\mathrm{RD}}$ is the reduced mobility of positive ion at standard condition $\left(\mathrm{T}_{\mathrm{gas}}=273 \mathrm{~K}\right.$ and $\mathrm{P}=1.103105 \mathrm{~Pa}$ ). The ionic mobility has been nearly constant at fixed ambient temperature, which is acceptable because the ambient temperature has been assumed not to change considerably compared to electron temperature. However, in a more accurate model, especially for hard and hot plasma, where temperatures become closer, the average of the ionic collision term must have been considered, too.

At the final step, the recombination rate has been included in relation (2) to examine the effect of changing of $\boldsymbol{D . I}$, through the recombination process, on the diagram of $T_{e}$ versus NR. For calculation the recombination (irradiative) rate, the following formulas based on the Thomson theory for recombination cross-sections [22-24] have been used to calculate mixed gas recombination rate versus $T_{e}$ in different media and gas temperature:

$$
\begin{aligned}
& \boldsymbol{\sigma}^{\boldsymbol{r e c}}=\boldsymbol{\pi}\left(\boldsymbol{k} \boldsymbol{e}^{2} /\left(\boldsymbol{E}_{\boldsymbol{e}}+\overline{\boldsymbol{E}}_{\boldsymbol{g}}\right)\right)^{2} \\
& \boldsymbol{\gamma}=\left\langle\boldsymbol{\sigma}^{\boldsymbol{r} \boldsymbol{c}} \boldsymbol{v}_{\boldsymbol{e}}\right\rangle
\end{aligned}
$$

where in these equations $\mathrm{k}$, and e are electrostatic constants and electric unit charges; $E_{e}$ and $\overline{E_{g}}$ are average thermal energies of electrons, and media, $\boldsymbol{\sigma}^{r e c}$, and $\boldsymbol{v}_{\boldsymbol{e}}$ are Thomson recombination crosssection, and electron velocity, respectively. It is obvious that in the non-equilibrium condition of the glow discharge, the change of $\mathrm{T}_{\text {gas }}$ at low electron temperatures exert very little influence on recombination rate and at high electron temperatures, especially in the range of $\mathrm{T}_{\mathrm{e}}>1^{\mathrm{ev}}, \mathrm{T}_{\mathrm{gas}}$ has no considerable effect on recombination.

\section{Results and discussion}

The diagrams of electron temperature versus similarity variable (NR) for $\mathrm{CO}_{2}, \mathrm{~N}_{2}$, and Helium have been derived using average electron density as a parameter in Figure 2, a, b, c, and the relation of $\mathrm{T}_{\mathrm{e}}$ and NR for three of them as a comparison has been shown in Figure $2 d$.
Although the dependence of $T_{e}$ on NR seems the same in this three gas, nevertheless the Figure $2 d$ shows that in a specified NR, the $\mathrm{T}_{\mathrm{e}}$ obeys a rule as $\mathrm{He}>\mathrm{N}_{2}>\mathrm{CO}_{2}$ that the electron temperature of the mixture can be controlled by changing ratio of $\mathrm{He}$, $\mathrm{N} 2$ and $\mathrm{CO} 2$ in gas mixture (it has to be considered also there are some other rules for $\mathrm{He}$, versus $\mathrm{N}_{2}$ and $\mathrm{CO}_{2}$, in the operating conditions of the $\mathrm{CO}_{2}$ laser, like arc suppression and thermal conduction). Also, it has to be cleared that up to, $D . I=10^{-6}$ no significant effect on the characteristic curve has been observed, which may be related to the simplicity of the model. At a higher degree of ionization, and electron density, the model has no physical result. The main reason for being limited to higher electron temperature with increasing the $n_{e}$ is that the effect of the other ionizing phenomena in pure media such as; photoionization and electron impact ionization of metastable states, which have been ignored here. These terms, where are hard to calculate, seem to be responsible for having a stable glow discharge at lower electron temperature accompanied by higher electron density $\left(\mathrm{n}_{\mathrm{e}}>10^{21} \mathrm{~m}^{-3}\right)$, which is related to D.I $>10^{-4}$. It should be mentioned that the radial average of electron density of the positive column, is concerning the axial value $\left(\mathrm{n}_{\mathrm{e}}\right)_{\max }$ as follows:

$$
\begin{aligned}
& n_{e}(r)=n_{e}{ }^{\max } J\left(\frac{2.4}{R} r\right) \text { Where } \\
& \overline{n_{e}}=\frac{1}{R} \int_{0}^{R} n_{e}\left(r^{\prime}\right) d r^{\prime} \& n_{e}{ }^{\max }=2.6 * \bar{n}_{e}
\end{aligned}
$$

It means that in a glow discharge with cylindrical geometry, $n_{e}{ }^{\max }$ and $\overline{n_{e}}$ have the same order of magnitude, and the reported experimental values almost agree with this statement [25]. It should be noted that there is a difference between operations in sealed off and gas flow regimes. In a sealed tube, by increasing of ambient temperature, the total density remains constant, but the pressure will rise, so the NR is a more suitable parameter for controlling the $T_{e}$. On the contrary, under gas flow condition especially with high gas flow rate, when ambient temperature goes high, the pressure at each point remains constant; a value between the input and exhaust pressure of the tube, but total mixture density will decrease, so the (input) pressure (P) multiplied by tube radius $(\mathrm{R})$ will be a useful similarity variable to adjust the $T_{e}$ for optimum energy transfer from the electrons to the excitation levels of $\mathrm{CO}_{2}$ and $\mathrm{N}_{2}$ molecules in glow discharge. 


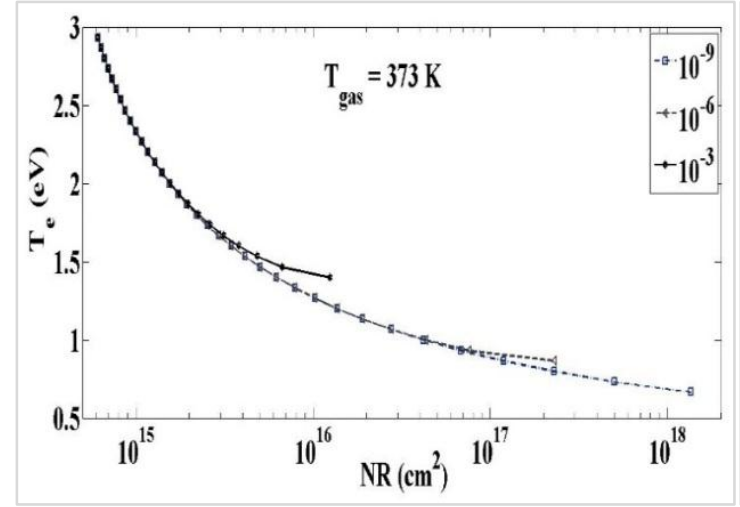

(a)

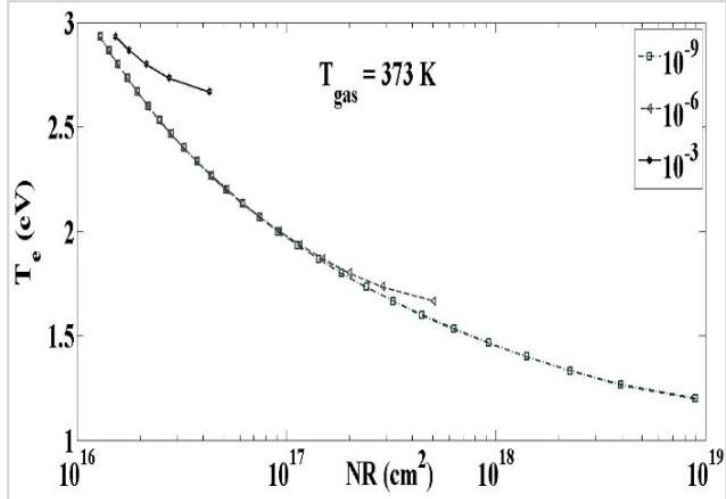

(c)

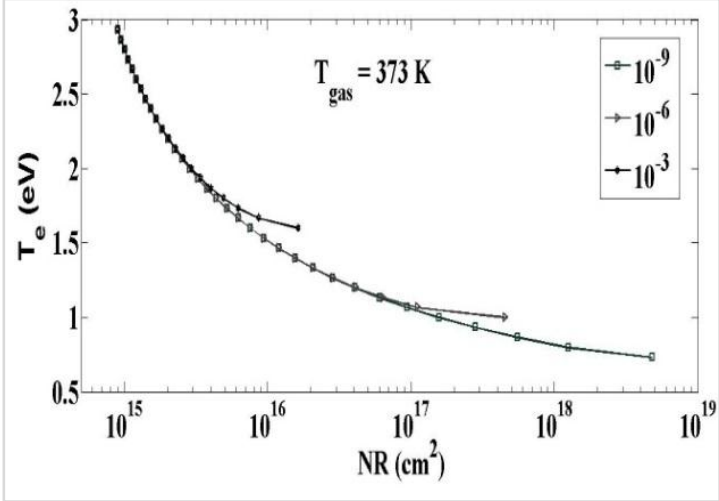

(b)

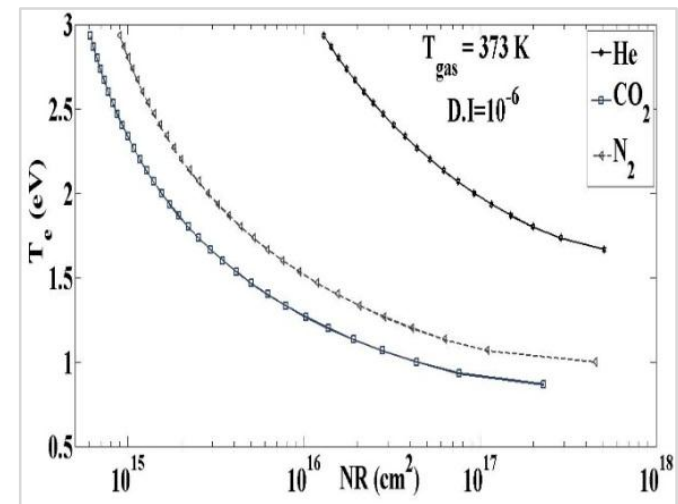

(d)

Figure $2 \mathrm{~T}_{\mathrm{e}}$ vs. NR with I.R as a parameter for different gases with three D.I (a) $\mathrm{CO}_{2}$ (b) $\mathrm{N}_{2}$ (c) $\mathrm{He}$ and (d) all of three gases in fixed $\mathrm{T}_{\mathrm{g}}$ and D.I.

\section{Conclusion}

From the diffusion-dominated time-independent glow discharge with cylindrical geometry, the characteristic electron energy (KTe) has been derived, which relates the (radial average) electron temperature to the similarity variable (NR). In this work, the ionization rates of $\mathrm{CO}_{2}, \mathrm{~N}_{2}$, and $\mathrm{He}$ calculated by fitting an empirical formula to the experimental data have been used to calculate the ionization rates. Then, using the experimental data and a simple model, the reduced ionic motilities in the standard condition have been included in the model. The characteristic curves for three gases in the room temperature with three different degrees of ionization have been derived and for comparison shown in Figure 2. The effect of the gas kind on the $\mathrm{T}_{\mathrm{e}}$, in constant $\mathrm{NR}$, has been noted, and the possibility of controlling the $\mathrm{T}_{\mathrm{e}}$ by mixing these gases, as it is done in the $\mathrm{CO}_{2}$ laser, have been discussed. The difference of the gas flow and gas sealed of glow discharge vessels respect to NR has been explained, too. In future, the extension of this method to the mixture of gases would be in the schedule.

Acknowledgment

None.

\section{Conflicts of interest}

The authors have no conflicts of interest to declare.

\section{References}

[1] Smirnov BM. Theory of gas discharge plasma. Cham: Springer International Publishing; 2015.

[2] Raizer YP, Allen JE. Gas discharge physics. Berlin: Springer; 1997.

[3] Cherrington BE. Gaseous electronics and gas lasers. Elsevier; 2014.

[4] Verdeyen JT. Laser electronics. lael. 1989.

[5] Powell J. CO2 laser cutting. London: Springer-Verlag; 1993.

[6] Bogaerts A, Gijbels R. Fundamental aspects and applications of glow discharge spectrometric techniques. Spectrochimica Acta Part B: Atomic Spectroscopy. 1998; 53(1):1-42.

[7] Bogaerts A, Neyts E, Gijbels R, Van der Mullen J. Gas discharge plasmas and their applications. Spectrochimica Acta Part B: Atomic Spectroscopy. 2002; 57(4):609-58. 
[8] Sremački I, Gromov M, Leys C, Morent R, Snyders R, Nikiforov A. An atmospheric pressure non-selfsustained glow discharge in between metal/metal and metal/liquid electrodes. Plasma Processes and Polymers. 2020; 17(6):1900191.

[9] Demin NA, Fedoseev AV. Simulations of the glow discharge positive column parameters in helium. In Journal of Physics: Conference Series 2019 (p. 012151). IOP Publishing.

[10] Bokhan PA, Gugin PP, Zakrevsky DE, Lavrukhin MA. Study of the properties of an anomalous glow discharge generating electron beams in helium, oxygen, and Nitrogen. Plasma Physics Reports. 2019; 45(11):1035-52.

[11] Aram, M., Morshedian N, and Behrouzinia S. Analysis of the glow discharge in a $\mathrm{CW}-\mathrm{CO} 2$ laser based on the similarity variable diagrams. Open Access Journal of Physics. 2019. 3(3): 21-4.

[12] Yuan C, Kudryavtsev AA, Demidov VI. Introduction to the kinetics of glow discharges. Morgan \& Claypool Publishers; 2018.

[13] Meek JM, Craggs JD. Electrical breakdown of gases.1978.

[14] Colonna G. Boltzmann and vlasov equations in plasma physics Plasma Modeling. 2016.

[15] Kumar M, Khare J, Nath AK. Numerical solution of Boltzmann tranport equation for TEA CO2 laser having nitrogen-lean gas mixtures to predict laser characteristics and gas lifetime. Optics \& Laser Technology. 2007; 39(1):86-93.

[16] Aram M, Morshedian N, Behrouzinia S, Namnabat M. An Innovative Simple Method for Study of the Characteristics of the Trigatron Plasma Switch. Contributions to Plasma Physics. 2016; 56(10):982-6.
[17] Kim YK, Irikura KK, Rudd ME, Ali MA, Stone PM, Coursey JS, et al. Electron-impact cross sections for ionization and excitation. NIST Standard Reference Database. 2010.

[18] McDaniel EW, Mason EA. Mobility and diffusion of ions in gases. 1973.

[19] Coxon P, Moruzzi J. Positive ion mobilities in carbon dioxide. Journal de Physique Colloques.1979.

[20] Moseley JT, Snuggs RM, Martin DW, McDaniel EW. Mobilities, diffusion coefficients, and reaction rates of mass-indentified nitrogen ions in nitrogen. Physical Review. 1969; 178(1).

[21] Beaty EC, Patterson PL. Mobilities and reaction rates of ions in helium. Physical Review. 1965; 137(2A): A346.

[22] Bates DR. Classical theory of electron-ion recombination in an ambient gas. Journal of Physics B: Atomic and Molecular Physics. 1980; 13(13).

[23] Center RE. Electron-ion recombination measurements in $\mathrm{CO}$ at high pressures. Journal of Applied Physics. 1973; 44(8):3538-42.

[24] Ernst GJ, Boer AG. Experimental determination of the electron-avalanche and the electron-ion recombination coefficient. Optics Communications. 1980; 34(2):2359.

[25] Baranov VY, Ul'Yanov KN. Contraction of a positive column. I*. Soviet Physics. Technical Physics. 1969; 14:121-4. 